\title{
Antimicrobial Resistance in Urinary Isolates of Escherichia coli with Special Reference to Fluoroquinolone Resistance and Extended Spectrum Beta-Lactamase (ESBL) Production
}

\author{
Jyoti Rajowar ${ }^{1}$, Sangeeta Dey Akoijam², Aninda Sen ${ }^{3}$, Kahkashan Akhter ${ }^{4}$, Shreshy Singh ${ }^{5}$ \\ 1,2,3,4,5 Department of Microbiology, Katihar Medical College, Bihar, India.
}

\section{ABSTRACT}

\section{BACKGROUND}

The commonest bacterial agent involved in causation of urinary tract infection (UTI) is Escherichia coli, both in the community as well as in the hospital. In this study Escherichia coli strains isolated from patients with UTI were studied especially for extended spectrum beta-lactamase (ESBL) production and determination of fluoroquinolone resistance.

\section{METHODS}

This descriptive study was conducted in the Department of Microbiology, Katihar Medical College and Hospital from December 2018 to May 2020. Urine samples from suspected UTI cases were processed and bacterial isolates were identified as per standard protocol. Antimicrobial susceptibility testing was done by Kirby-Bauer discdiffusion method on Mueller-Hinton agar. ESBL detection was done as per Clinical and Laboratory Standards Institute (CLSI) guidelines.

\section{RESULTS}

Out of 3938 urine samples received in the microbiology laboratory, 708 samples showed significant growth of various bacteria and candida species, out of these only 105 patients had urinary tract infection caused by Escherichia coli. The male to female ratio was $0.25: 1$. Isolates were highly sensitive to nitrofurantoin $(80.9 \%)$ followed by amikacin (72.4\%) and imipenem (71.5\%). Maximum resistance was seen with amoxicillin (98.1\%), cefuroxime (96.2\%), cefpodoxime and cefotaxime (90.5\%), ceftriaxone (85.7\%), nalidixic acid (91.4\%) and ciprofloxacin (70.5\%). $70.5 \%$ were found to be ESBL producers and $29.5 \%$ were non-ESBL producers. The double disc synergy test (DDST) could detect only $42.8 \%$ of ESBL producers whereas phenotypic confirmatory disc diffusion (PCDDT) detected $70.5 \%$ of ESBL producers.

\section{CONCLUSIONS}

It was seen in the present study that a high proportion of community acquired strains of Escherichia coli were ESBL producers. In this study, $70.5 \%$ of Escherichia coli strains were ESBL positive. It can therefore be recommended that all gram-negative isolates be tested for ESBL production preferably by the PCDDT test as this test was found to be most sensitive for detection of ESBL production. The PCDDT test requires minimum laboratory infrastructure, is cheap and easy as compared to molecular methods.

\section{KEY WORDS}

Escherichia coli, UTI, ESBL, PCDDT, DDST
Corresponding Author: Dr. Jyoti Rajowar,

Room No. 101, Girl's P.G. Hostel, KMC Campus, Katihar Medical College, Karimbagh, Katihar,

Bihar - 854105, India.

E-mail:

jyotirajowarpoddar777@gmail.com

DOI: 10.14260/jemds/2021/126

How to Cite This Article:

Rajowar J, Akoijam SD, Sen A, et al. Antimicrobial resistance in urinary isolates of Escherichia coli with special reference to fluoroquinolone resistance and extended spectrum beta-lactamases (esbl) production. J Evolution Med Dent Sci 2021;10(09):583-588, DOI: 10.14260/jemds/2021/126

Submission 24-10-2020,

Peer Review 02-01-2021,

Acceptance 09-01-2021,

Published 01-03-2021.

Copyright (C) 2021 Jyoti Rajowar et al. This is an open access article distributed under Creative Commons Attribution License [Attribution 4.0 International (CC BY 4.0)] 


\section{BACKGROUND}

Urinary tract infections (UTI) imply invasion of urinary tract by pathogens, which may involve the upper or lower tract depending on whether the infection is in the kidney or bladder and urethra. ${ }^{1}$ Lower UTIs may affect the urethra or the bladder. In upper UTI the ureters or the renal parenchyma are affected.2

It is a common condition that occurs in both males and females of all ages. The prevalence and incidence of infection are higher in women than in men which are likely the result of several clinical factors including anatomical differences, hormonal effects and behaviour patterns. ${ }^{3}$ The commonest bacterial agent causing UTI is Escherichia coli, both in the community as well as in the hospital. ${ }^{4}$ Escherichia coli cause approximately $80 \%$ of acute infections in patients without catheters, urologic abnormalities or calculi. The prevalence of antimicrobial resistance among the human clinical isolates of Escherichia coli has increased dramatically in the recent years. ${ }^{5}$

Urinary pathogens have shown a changed pattern of susceptibility to antibiotics, resulting in an increase in resistance to commonly used antibiotics. ${ }^{4}$ Over the past decade, fluoroquinolone resistance rates have increased to > $10 \%$ in some surveys. In many parts of the world, Escherichia coli fluoroquinolone resistance rates are > $20 \%$ among patients with community acquired uncomplicated UTI and > $50 \%$ among patients with complicated infections. ${ }^{6}$ Resistance to fluoroquinolone is caused by mutations in the chromosomal genes that code the DNA gyrase and / or DNA topoisomerase IV, which are the target enzymes, resulting in alteration in drug accumulation. ${ }^{7}$

It is seen that fluoroquinolone resistance among the urinary isolates is also associated with multidrug resistance. ${ }^{8}$ MDR is defined as resistance to at least two antibiotics of different classes including aminoglycosides, chloramphenicol, tetracyclines and / or erythromycin. MDR in many bacteria is due to the action of multi drug efflux pumps and by the accumulation of resistance $®$ plasmids or transposons with each coding for resistance to a specific agent. 9

The first report of plasmid encoded $\beta$-lactamases capable of hydrolysing the extended spectrum cephalosporins was published in 1983. The gene encoding the $\beta$-lactamase showed a mutation of a single nucleotide compared to the gene encoding SHV-1. Other $\beta$-lactamases were soon discovered which were closely related to TEM-1 and TEM-2, but which had the ability to confer resistance to the extended-spectrum cephalosporins. Hence these new $\beta$-lactamases were coined extended-spectrum $\beta$-lactamases (ESBLs). Extended spectrum $\beta$-lactamases are $\beta$-lactamases capable of conferring bacterial resistance to the penicillins, first, second, and third generation cephalosporins, and aztreonam (but not the cephamycins or carbapenems) by hydrolysis of these antibiotics, and which are inhibited by $\beta$-lactamase inhibitors such as clavulanic acid. ${ }^{10}$

The prevalence of ESBL positive isolates depends on a range of factors including species, geographic location, hospital / ward, group of patients and type of infection, and large variations have been reported in different studies. Delayed reporting of ESBL producing gram-negative bacilli is associated with prolonged hospital stay, increased morbidity and high health care costs. ${ }^{3}$ So that is why accurate identification of pathogens with short turnaround time and generation of authenticated results is the primary responsibility of a clinical microbiology laboratory. In this study Escherichia coli strains isolated from patients with UTI were studied especially for ESBL production and fluoroquinolone resistance.

\section{METHODS}

All patients attending the out-patient department and admitted in various wards in Katihar Medical College Hospital, with signs and symptoms of UTI were inducted into the study that was conducted from December 2018 to May 2020. Institutional ethical committee clearance was obtained and informed consent was obtained from each and every patient. Early morning midstream clean catch urine was collected in a sterile, wide mouthed, screw capped leak proof universal container. Samples that were not collected according to instructions given to the patient were excluded from the study.

Samples were transported to the laboratory without delay. Microscopical examination of urine was done by wet film preparation. A standard loop (No. 24 SWG) measuring $4 \mathrm{~mm}$ in internal diameter and with a holding capacity of $0.01 \mathrm{ml}$ of urine was employed for culture. Urine was streaked on three petri dishes having blood agar, cysteine lactose electrolyte deficient agar and MacConkey's agar using standard culture techniques. ${ }^{11}$ After inoculation, plates were incubated at $37^{\circ} \mathrm{C}$ for $18-24$ hours. Bacterial colonies on the culture media were counted for quantification of bacterial growth and processed for their identification based on colony morphology, Gram stain findings, motility and biochemical tests.

All Escherichia coli isolates were tested against different antimicrobial agents on Mueller-Hinton agar plates by KirbyBauer disc-diffusion method. Antimicrobial agents tested were amoxicillin, gentamicin, cotrimoxazole, nitrofurantoin, amikacin, ciprofloxacin, cefixime, cefuroxime, cefotaxime, cefpodoxime, ceftriaxone, cefoperazone / sulbactum and imipenem. Escherichia coli ATCC 25922 was used as a standard strain for routine quality control.

The isolates which were resistant to ciprofloxacin were tested against fluoroquinolones of each generation as a surrogate marker for increased generations. The following fluoroquinolones were used for further testing: first generation-ciprofloxacin (5 mcg), second generationlevofloxacin ( $5 \mathrm{mcg}$ ), fourth generation-moxifloxacin $(5 \mathrm{mcg}$ ). The results of antibiotic susceptibility testing were interpreted by zone interpretation guidelines as per recommendations of CLSI. ${ }^{12}$

Recommended guidelines for screening of ESBL Cefotaxime zone $\leq 27 \mathrm{~mm}$

Cefpodoxime zone $\leq 17 \mathrm{~mm}$ Ceftriaxone zone $\leq 25 \mathrm{~mm}$

If the zone diameter of any isolate for any one of the antibiotics was less than or equal to the zone sizes shown above, the isolate was further tested by the confirmatory test for ESBL production

\section{Double Disc Synergy Test (DDST)}

Bacterial isolate to be tested was prepared in Mueller-Hinton broth (MHB). After matching its turbidity to 0.5 McFarland's 
standard, streaking was done over the entire Mueller-Hinton agar (MHA) plate as per guidelines for disc diffusion technique. Amoxycillin / clavulanic acid disc was placed in the centre of the plate. Antibiotic discs of cefotaxime, ceftazidime, ceftriaxone and cefpodoxime were placed at a distance of 20 $\mathrm{mm}$ (centre to centre) from amoxicillin / clavulanic acid disc and at 90 degree from each other. The plate was then incubated overnight at $37^{\circ} \mathrm{C}$. ESBL production was suggested by the enhancement of inhibition zone for any of the four antibiotic discs towards amoxicillin / clavulanic acid disc. Isolates which showed enhancement of inhibition zone were further confirmed by CLSI recommended phenotypic confirmatory test.

\section{Phenotypic Confirmatory Disc Diffusion Test (PCDDT)}

Bacterial isolate to be tested was prepared in MHB. After matching its turbidity to 0.5 McFarland's standard, the organism was inoculated over the entire MHA surface as per guidelines for disc diffusion technique. Antibiotic discs of cefotaxime, cefotaxime / clavulanic acid, ceftazidime, and ceftazidime / clavulanic acid were placed at a distance of 30 $\mathrm{mm}$ (centre to centre) from each other. The plate was then incubated overnight at $37^{\circ} \mathrm{C}$. ESBL production was confirmed by a difference of $\geq 5 \mathrm{~mm}$ between the inhibition zone of either third generation cephalosporin disc and their respective clavulanic acid combination disc.

\section{Statistical Analysis}

Statistical analysis was carried out using online software www.physics.csbsju.edu / cgi-bin / stats / contingency accessed on 21.08.2020. $\mathrm{P}$ value was calculated using chisquare test, $P$ value $\leq 0.05$ was considered to be significant and $P$ value $\leq 0.001$ was considered to be highly significant.
RESULTS

A total of 3938 urine samples were received in the laboratory from December 2018 to May 2020 out of which 3105 samples showed no growth, 125 samples showed insignificant bacteriuria. A total of 708 samples showed growth of various bacteria and candida species. Out of these 708 isolates, 105 patients had urinary tract infection caused by Escherichia coli. These isolates were subjected to antibiotic susceptibility testing, ESBL screening and phenotypic cofirmatory tests.

Out of the 105 Escherichia coli isolates, 84 were from female and 21 were from male patients. The male to female ratio was $0.25: 1$, this finding was found to be statistically highly significant $(P=0.000)$. Maximum number of cases were seen in the age group of $0-10$ years $(25.7 \%)$ followed by the age group of $21-30$ years (23.8\%). Majority of the patients were from OPD $60.9 \%$ and rest of the patients were from IPD $39.1 \%$. Antibiotic susceptibility pattern showed most of the strains to be susceptible to nitrofurantoin $80.9 \%$ followed by amikacin $72.4 \%$ and imipenem $71.5 \%$. Most of the isolates were resistant to amoxicillin $98.1 \%$ followed by cefuroxime $96.2 \%$. Resistance to $3^{\text {rd }}$ generation cephalosporins was also substantial being $90.5 \%$ for cefpodoxime and cefotaxime and $85.7 \%$ for ceftriaxone and cefixime. Figure 1 shows antimicrobial resistance pattern of Escherichia coli.

Among the quinolones and fluoroquinolones tested, maximum resistance was seen to nalidixic acid $91.4 \%$ followed by ciprofloxacin $70.5 \%$, ofloxacin $57.2 \%$, levofloxacin $49.5 \%$ and moxifloxacin $47.6 \%$. Table 1.

ESBL production was found in $70.5 \%$ of Escherichia coli isolates and $29.5 \%$ were non-ESBL producers. Maximum number of cases were seen in the age group of $21-30$ years $(24.3 \%)$ followed by age group of $41-50$ years (18.9\%).

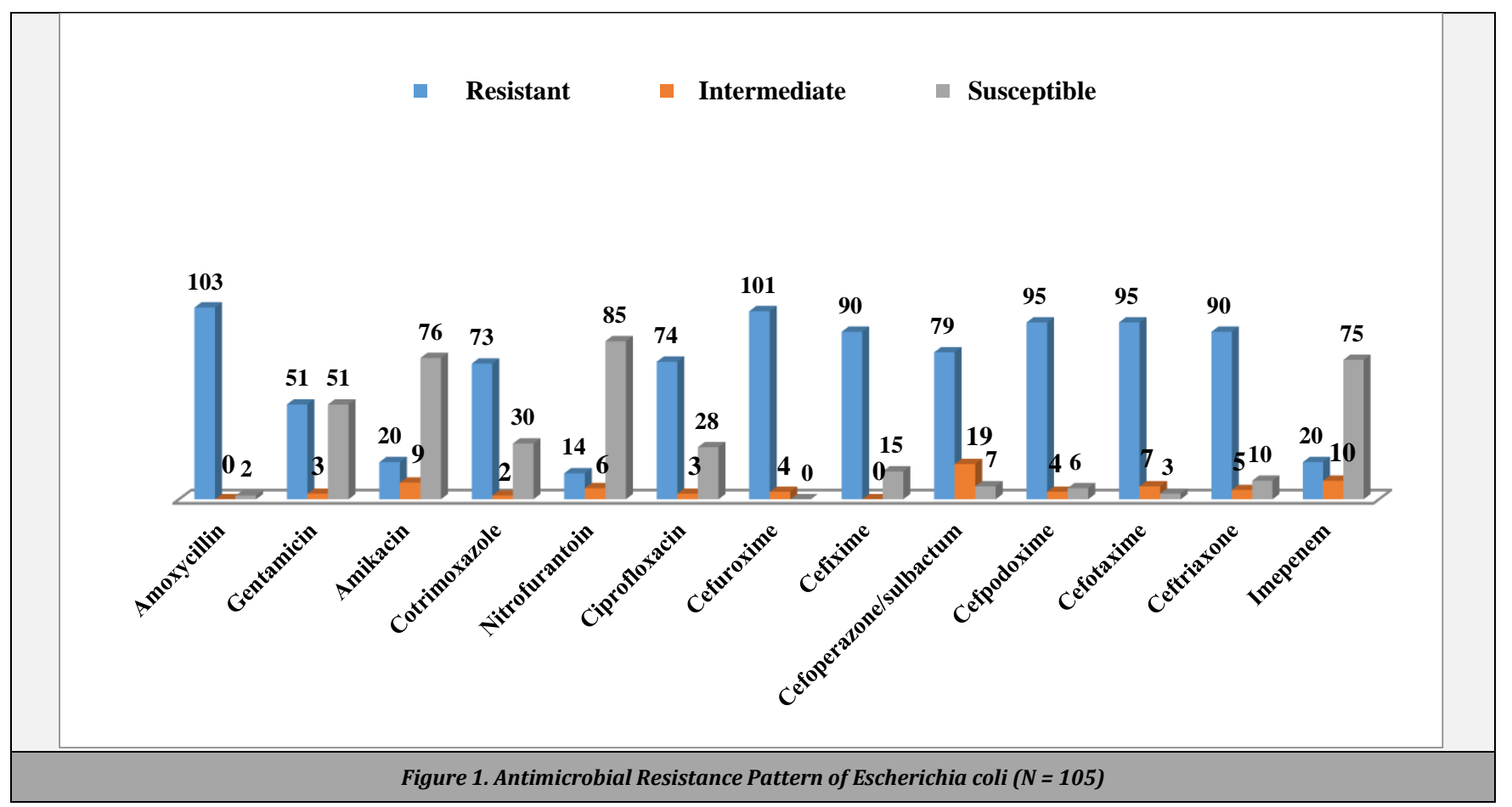


Out of a total of 74 ESBL producers, rate of isolation was seen more in females (79.7 \%) than males (20.3\%). Majority of ESBL producing Escherichia coli isolates were from IPD 51.4 $\%$ while $48.6 \%$ were from OPD.

By the ESBL screening test all isolates of Escherichia coli were found to be ESBL producers when the strains were tested against cefotaxime and ceftriaxone by following the CLSI criteria. When tested against cefpodoxime, $90.5 \%$ strains were found to be ESBL producers. By the DDST only $42.8 \%$ were found to be ESBL producers whereas by the PCDDT which was taken as the confirmatory test $70.5 \%$ of strains were found to be positive as shown in Table 2 .

Among the ESBL producers, resistance to cefpodoxime was maximum $98.6 \%$ followed by cefuroxime $97.3 \%$ and cefotaxime and amoxicillin $95.9 \%$ each as shown in Table 3. $81.1 \%$ of isolates were sensitive to nitrofurantoin, followed by amikacin $70.3 \%$, and $67.6 \%$ each to imipenem and gentamicin. Among the non-ESBL producers, all isolates were resistant to amoxicillin followed by cefuroxime $93.5 \%$ and cefotaxime $77.4 \%$. Nitrofurantoin and imipenem were two antibiotics to which $80.6 \%$ of Escherichia coli isolates were sensitive followed by amikacin $74.2 \%$.

\begin{tabular}{|cccc|}
\hline $\begin{array}{c}\text { Quinolones and } \\
\text { Fluoroquinolones }\end{array}$ & $\begin{array}{c}\text { Resistant } \\
\text { (\%) }\end{array}$ & $\begin{array}{c}\text { Intermediate } \\
\text { (\%) }\end{array}$ & $\begin{array}{c}\text { Sensitive } \\
\text { (\%) }\end{array}$ \\
Nalidixic acid & $96(91.4)$ & $3(02.9)$ & $6(05.7)$ \\
Ciprofloxacin & $74(70.5)$ & $3(02.9)$ & $28(26.6)$ \\
Ofloxacin & $60(57.2)$ & $4(03.8)$ & $41(39.0)$ \\
Levofloxacin & $52(49.5)$ & $6(05.7)$ & $47(44.8)$ \\
Moxifloxacin & $50(47.6)$ & $5(04.8)$ & $50(47.6)$ \\
\hline \multicolumn{4}{|c|}{ Table 1. Quinolone and Fluoroquinolone Resistance } \\
Pattern of Escherichia coli (N = 105) \\
\hline \multicolumn{4}{c}{} \\
\hline
\end{tabular}

\begin{tabular}{|cccc|}
\hline Method & Positive (\%) & Negative (\%) & Total \\
\hline ESBL screening test & $95(90.5)$ & $10(09.5)$ & $\mathbf{1 0 5}$ \\
DDST & $45(42.8)$ & $60(57.1)$ & $\mathbf{1 0 5}$ \\
PCDDT & $74(70.5)$ & $31(29.5)$ & $\mathbf{1 0 5}$ \\
\hline \multicolumn{2}{|c|}{ Table 2. Comparative Study of Different Methods of ESBL Detection } \\
\hline
\end{tabular}

\begin{tabular}{|c|c|c|c|c|c|c|}
\hline \multirow{3}{*}{ Antibiotic } & \multirow{2}{*}{\multicolumn{3}{|c|}{$\begin{array}{c}\text { ESBL Producer }(\mathrm{N}=74) \\
\text { Sensitiv Intermediat Resista }\end{array}$}} & \multicolumn{3}{|c|}{ Non-ESBL Producer $(\mathrm{N}=31)$} \\
\hline & & & & e & e & nt \\
\hline & $\%$ & $\%$ & $\%$ & $\%$ & $\%$ & $\%$ \\
\hline Amoxycillin & 04.1 & 0 & 95.9 & 0 & 0 & 100 \\
\hline Amikacin & 70.3 & 08.1 & 21.6 & 74.2 & 09.7 & 16.1 \\
\hline Gentamicin & 67.6 & 02.7 & 29.7 & 64.5 & 03.2 & 32.3 \\
\hline $\begin{array}{c}\text { Cotrimoxazol } \\
\mathrm{e}\end{array}$ & 24.3 & 02.7 & 73.0 & 38.7 & 0 & 61.3 \\
\hline Ciprofloxacin & 24.3 & 02.7 & 73.0 & 32.3 & 03.2 & 64.5 \\
\hline $\begin{array}{c}\text { Nitrofurantoi } \\
\mathrm{n}\end{array}$ & 81.1 & 02.7 & 16.2 & 80.6 & 12.9 & 06.5 \\
\hline Cefuroxime & 0 & 02.7 & 97.3 & 0 & 06.5 & 93.5 \\
\hline Cefixime & 06.8 & 0 & 93.2 & 32.3 & 0 & 67.7 \\
\hline Cefpodoxime & 0 & 01.4 & 98.6 & 19.3 & 09.7 & 71.0 \\
\hline Cefotaxime & 01.4 & 02.7 & 95.9 & 06.5 & 16.1 & 77.4 \\
\hline Ceftriaxone & 04.1 & 04.1 & 91.8 & 22.6 & 06.4 & 71.0 \\
\hline Imipenem & 67.6 & 06.7 & 25.7 & 80.6 & 16.1 & 03.2 \\
\hline
\end{tabular}

\section{DISCUSSION}

In the present study, there was predominance of female patients, $80 \%$ of patients with UTI being female. The male to female ratio was $0.25: 1$ which was found to be statistically highly significant $(\mathrm{P}=0.000)$. Other studies have also reported a male to female ratio of 1:2.2 and 0.5:1.3,13 Maximum number of patients in this study were from the age group of 0 - 10 years (25.7\%) followed by 21 - 30 years $(23.8 \%)$.
Other workers have reported maximum number of cases in the age group of 20 - 40 years (46.03\%). ${ }^{9}$ This finding is different from the findings of the present study.

Out of the 105 patients, majority of the patients were from OPD $60.9 \%$ and rest of them were from IPD $39.1 \%$, this finding was found to be statistically insignificant $(\mathrm{P}=0.066)$. The outdoor to indoor ratio was 1.6:1. Similar findings have been reported by others. ${ }^{3,14}$ This could be explained by the fact that UTI per se, especially lower UTI, does not warrant hospitalisation and can be effectively treated as outdoor cases. Majority of the indoor cases with UTI were hospital acquired and were not admitted for the treatment of this disorder.

Maximum resistance was seen with amoxicillin $98.1 \%$ followed by cefuroxime $96.2 \%$. Most of the organisms were sensitive to nitrofurantoin $80.9 \% .72 .4 \%$ of organisms were sensitive to amikacin and $71.5 \%$ to imipenem. Studies with similar findings have been reported. ${ }^{14,15}$

In this study, $91.4 \%$ of Escherichia coli strains were resistant to nalidixic acid and $70.5 \%$ to ciprofloxacin. Similar resistance pattern were seen in the study done by Nandihal 2015. ${ }^{7}$

It was noted that with the use of higher generations of fluoroquinolones, the percentage of resistance also reduced e.g. $70.5 \%$ of Escherichia coli were resistant to ciprofloxacin (first generation), $49.5 \%$ to levofloxacin (second generation) and $47.6 \%$ to moxifloxacin (fourth generation). Gururaju et al. in 2015 also reported slight decrease in resistance rates with the use of higher generations of fluoroquinolones. ${ }^{4}$

It was found that $70.5 \%$ of Escherichia coli isolates causing UTI were ESBL producers as compared to $29.5 \%$ that did not produce ESBLs. This finding was found to be statistically highly significant $(\mathrm{P}=0.001)$. Bhandari et al. in 2016 reported that $72.3 \%$ of their Escherichia coli isolates were ESBL producers. ${ }^{16}$ Other authors have reported slightly higher rate of ESBL production i.e. $76.5 \%$ in Escherichia coli isolates. ${ }^{17}$ In this study maximum number of ESBL producing Escherichia coli was from the age group of 21 - 30 years followed by 41 50 years and least in $61-70$ years. This is similar to study conducted by Shakya et al 2017.3 Another study conducted in 2009 however, reported maximum number of ESBL producing Escherichia coli isolates in the age group of $30-39$ years 32.2 $\% .{ }^{18}$

ESBL producing isolates were more in females $79.7 \%$ than in males $20.3 \%$. This is probably due to the fact that majority of patients included in this study were females. This difference was found to be statistically highly significant $(\mathrm{P}=0.000)$. Similar findings have also been reported by other workers. ${ }^{15,17}$

The number of ESBL producing Escherichia coli was more in indoor patients (51.4\%) as compared to outdoor patients (48.6\%). Similar studies were done by Rahman et al. in 2018 who reported that $58.07 \%$ of ESBL producing isolates were from IPD and $41.93 \%$ were from OPD. ${ }^{14}$

Out of the 105 isolates which were tested, $70.5 \%$ were found to be ESBL producers by PCDDT and $42.8 \%$ by DDST. DDST failed to detect ESBL production in $39.2 \%$ of Escherichia coli isolates. Taking PCDDT as the phenotypic cofirmatory test, the DDST was found to give a high number of false negatives. Similar reports were made by other workers however, the false negativity rate in their study was much less (4.7\%) as compared with that of the present study (39.2\%). ${ }^{19}$ The PCDDT was found to be a better test for detection of ESBL production. 
Our study reveals that most of the ESBL producing isolates were sensitive to nitrofurantoin $(81.1 \%)$ followed by amikacin (70.3\%), and imipenem (67.6 \%). Maximum resistance among ESBL producers was $98.6 \%$ to cefpodoxime followed by $97.3 \%$ to cefuroxime. Similar findings have been reported by others. ${ }^{19,} 20,21$

\section{CONCLUSIONS}

Quinolone and fluoroquinolone resistance was found to be fairly high in the present study, $91.4 \%$ of Escherichia coli strains were found to be resistant to nalidixic acid. Resistance to ciprofloxacin, ofloxacin and moxifloxacin was found to be $70.5 \%, 57.2 \%$ and $44.8 \%$ respectively. ESBL production in Escherichia coli isolates was found to be very high which was $70.5 \%$. A good proportion of Escherichia coli isolates from outdoor patients was also found to be ESBL producers (48.6 $\%)$ which was totally unexpected. However, in the present study it was seen that even those isolates from patients with community acquired UTI were resistant to majority of antibiotics against which they were tested.

It is therefore advisable to routinely perform culture and sensitivity in all cases of UTI instead of resorting to empirical treatment especially with higher antibiotics as this can lead to development and spread of resistant strains not only in the hospital but also at the community level. It was also noted in the study that a very high number of Escherichia coli strains were ESBL positive (70.5\%). Though beyond the scope of the present study, as other gram-negative bacilli were not tested for ESBL production, it can indirectly be inferred that many of the strains isolated from this region may harbour ESBL genes. It can therefore be recommended that all gram negative isolates be tested for ESBL production preferably by the PCDDT test as this test was found to be most sensitive for detection of ESBL production. The PCDDT test requires minimum laboratory infrastructure, it is cheap and very easy to perform as compared to molecular methods.

Data sharing statement provided by the authors is available with the full text of this article at jemds.com.

Financial or other competing interests: None.

Disclosure forms provided by the authors are available with the full text of this article at jemds.com.

\section{REFERENCES}

[1] Hari P, Srivastava RN. Urinary Tract Infections. In: Srivastava RN, Bagga A, eds. Pediatric Nephrology. $5^{\text {th }}$ edn. New Delhi: Jaypee Brothers Med Publishers 2011: p. 273300.

[2] Tille PM. Infections of the urinary tract. Bailey \& Scott's Diagnostic Microbiology. $14^{\text {th }}$ edn. St. Louis, Missouri: Elsevier 2017: p. 987-98.

[3] Shakya P, Shrestha D, Maharjan E, et al. ESBL Production among E. coli and Klebsiella spp. Causing urinary tract infection: a hospital based study. Open Microbiol J 2017;11:23-30.

[4] Gururaju T, Sarojamma V, Ramakrishna V. Prevalence and fluoroquinolone resistance pattern in escherichia coli isolates of urinary tract infection (UTI) patients. Journal of Krishna Institute of Medical Sciences University 2015;4(2):56-64.

[5] Shariff VAAR, Shenoy MS, Yadav T, et al. The antibiotic susceptibility patterns of uropathogenic escherichia coli, with special reference to the fluoroquinolones. J Clin Diagn Res 2013;7(6):1027-30.

[6] Talan DA, Takhar SS, Krishnadasan A, et al. Fluoroquinolone-resistant and extended-spectrum $\beta$ lactamase-producing escherichia coli infections in patients with pyelonephritis, United States. Emer Infect Dis 2016;22(9):1594-603.

[7] Nandihal NW. Profile of urinary tract infection and quinolone resistance among escherichia coli and klebsiella species isolates. Int J Curr Microbiol App Sci 2015;4(7):749-56.

[8] Arundathi HA, Koppad M, Halesh LH, et al. Coexistence of quinolone resistance and extended spectrum beta lactamase production in urinary isolates of escherichia coli-an emerging challenge to antimicrobial prescribing pattern. Indian J Microbiol Res 2016;3(4):359-62.

[9] Yadav K, Prakash S. Screening of ESBL producing multidrug resistant e. coli from urinary tract infection suspected cases in Southern Terai of Nepal. J Infect Dis Diagn 2017;2(2):116.

[10] Paterson DL, Bonomo RA. Extended-spectrum $\beta$ lactamases: a clinical update. Clin Microbiol Rev 2005;18(4):657-86.

[11] Collee JG, Marr W. Culture of bacteria. In: Collee JG, Fraser AG, Marmion BP, et al. eds. Mackie and McCartney Practical Medical Microbiology. 14th edn. Churchill Livingstone/ Elsevier 1996: p. 113-30.

[12] Performance Standards for Antimicrobial Susceptibility Testing. CLSI Supplement M100. 30th edn. Wayne, PA: Clinical and Laboratory Standards Institute 2020: p. 1402.

[13] FarajzadehSheikh A, Veisi H, Shahin M, et al. Frequency of quinolone resistance genes among extended-spectrum $\beta$ lactamase (ESBL)-producing escherichia coli strains isolated from urinary tract infections. Trop Med Health 2019;47:19.

[14] Rahman MS, Garg R, Singh VA, et al. Antibiotic susceptibility profile and extended spectrum $\beta$ lactamases production by uropathogenic escherichia coli from tertiary care hospital of rural settings. Int J Res Med Sci 2018;6(12):4022-7.

[15] Smita MS, Wavare SS, Gajul S, et al. Bacterial profile of urinary tract infections and antibiotic resistance pattern in a tertiary care hospital. Online J Health Allied Sci 2019;18(4):6.

[16] Bhandari R, Pant ND, Poudel A, et al. Assessment of the effectiveness of three different cephalosporin/ clavulanate combinations for the phenotypic confirmation of extended-spectrum beta-lactamase producing bacteria isolated from urine samples at National Public Health Laboratory, Kathmandu, Nepal. BMC Res Notes 2016;9:390.

[17] Abayneh M, Tesfaw G, Abdissa A. Isolation of extendedspectrum $\beta$-lactamase (ESBL-) producing Escherichia coli and Klebsiellapneumoniaefrom patients with community onset urinary tract infections in Jimma University 
Specialized hospital, Southwest Ethiopia. Can J Infect Dis Med Microbiol 2018;2018:4846159.

[18] Wani KA, Thakur MA, Fayaz AS, et al. Extended spectrum B-lactamase mediated resistance in escherichia coli in a tertiary care hospital. Int J Health Sci (Qassim) 2009;3(2):155-63.

[19] Dalela G. Prevalence of extended spectrum betalactamase (ESBL) producers among Gram-negative bacilli from various clinical isolates in a tertiary care hospital at Jhalawar, Rajasthan, India. Journal of Clinical and Diagnostic Research 2012;6(2):182-7.
[20] Gautam V, Thakur A, Sharma M, et al. Molecular characterization of extended-spectrum $\beta$-lactamases among clinical isolates of escherichia coli and klebsiella pneumoniae: a multi-centric study from tertiary care hospitals in India. Indian J Med Res 2019;149(2):208-15.

[21] Harwalkar A, Sataraddi J, Gupta S, et al. The detection of ESBL producing Escherichia coli in patients with symptomatic urinary tract infections using different diffusion methods in a rural setting. J Infect Public Health 2012;6(2):108-14. 